\title{
Existence and Uniqueness of Positive and Bounded Solutions of a Discrete Population Model with Fractional Dynamics
}

\author{
J. E. Macías-Díaz \\ Departamento de Matemáticas y Física, Universidad Autónoma de Aguascalientes, Avenida Universidad 940, \\ Ciudad Universitaria, 20131 Aguascalientes, AGS, Mexico \\ Correspondence should be addressed to J. E. Macías-Díaz; jemacias@correo.uaa.mx
}

Received 8 March 2017; Accepted 19 April 2017; Published 8 May 2017

Academic Editor: Douglas R. Anderson

Copyright (C) 2017 J. E. Macías-Díaz. This is an open access article distributed under the Creative Commons Attribution License, which permits unrestricted use, distribution, and reproduction in any medium, provided the original work is properly cited.

We depart from the well-known one-dimensional Fisher's equation from population dynamics and consider an extension of this model using Riesz fractional derivatives in space. Positive and bounded initial-boundary data are imposed on a closed and bounded domain, and a fully discrete form of this fractional initial-boundary-value problem is provided next using fractional centered differences. The fully discrete population model is implicit and linear, so a convenient vector representation is readily derived. Under suitable conditions, the matrix representing the implicit problem is an inverse-positive matrix. Using this fact, we establish that the discrete population model is capable of preserving the positivity and the boundedness of the discrete initial-boundary conditions. Moreover, the computational solubility of the discrete model is tackled in the closing remarks.

\section{Introduction}

The development in recent decades of fractional calculus has led to important discoveries in many scientific areas $[1,2]$. For example, research in the physical sciences has developed toward the construction of a physically meaningful calculus of variations for fractional systems [3, 4]. Here, the problem lies in the fact that the notions of "energy" and "Hamiltonian" still lack a concrete physical connotation, so the determination of the physical significance of those concepts is still a fruitful area of study [5]. In the field of differential/difference equations, the determination of theorems on the existence and the uniqueness of solutions of fractional systems is nowadays an area of analytic importance [6]. In this context, the determination of the properties of the relevant solutions of fractional systems is also a transited avenue of research, with the conditions of positivity and boundedness being of particular interest [7]. From a numerical perspective, the design of computational techniques with desirable numerical properties (convergence, stability, consistency, etc.) is also a scientific problem that has attracted the attention of researchers in the area [8]. However, the development of numerical methods that are capable of preserving structural properties of their continuous counterparts (the positivity, the boundedness, the monotonicity, or the preservation of energy, mass, momentum, etc.) is still scarce.

One of the most studied partial differential equations in the literature is the well-known diffusion-reaction model investigated simultaneously and independently in 1937 by Fisher [9] and Kolmogorov et al. [10]. That model possesses positive, bounded, and traveling-wave solutions, and it was used initially to describe the propagation of mutant genes that are advantageous to the survival of populations distributed in linear habitats [11]. Fisher's equation has also found applications in the investigation of the neutron flux and temperature in prompt feedback nuclear reactors [12], where the governing law is the Pearl-Verhulst equation. As many other equations from the physical sciences, that model has also been extended to the fractional scenario in order to describe more accurately the phenomena of interest (see [13] and the references therein). A vast number of criteria for fractional differentiation have been employed to that end, but some of these extensions are capable of resembling the properties of the solutions of the model investigated in 1937, like the existence and uniqueness of traveling-wave solutions that are positive and bounded [14].

On the other hand, the exact determination of solutions of fractional extensions of Fisher's equation is a difficult task. 
To solve this problem, various computational approaches have been designed in order to approximate the solutions of continuous (fractional or integral) Fisher's equations. Some approximation techniques have been proposed in the literature to that effect, including finite-difference schemes [15], differential and integral quadrature techniques [16, 17], Legendre spectral collocation methods [18], discrete local discontinuous Galerkin methods [19], finite volume schemes with preconditioned Lanczos techniques [20], and homotopy perturbation methods [21]. In most of the cases, the methods proposed are capable of approximating the solutions of the fractional Fisher's equation with a high degree of precision, but the preservation of the most important features of the solutions of interest is neglected. The present manuscript is motivated by a Riesz space-fractional extension of Fisher's equation for which traveling-wave solutions that are positive and bounded exist. Full discretization that uses fractional centered differences is considered in this work and we show that, as its continuous counterpart, the discrete model possesses solutions that are positive and bounded. More precisely, we provide a dynamically consistent fully discrete form of our fractional model in the spirit of many of the works by Mickens and coworkers [22].

The present note is sectioned as follows. In Section 2, we introduce the fractional partial differential equation that motivates our investigation. The model is an extension of Fisher's equation from population dynamics in which the diffusion term is expressed as a Riesz fractional derivative. Both the continuous and the discrete Riesz fractional operators are presented in that section, together with some useful lemmas quoted from the standard literature. The discrete fractional Fisher's model is presented in Section 3. The discrete nomenclature and pertinent initial-boundary conditions are given therein as well as a vector form of the discrete model. In Section 4, we establish the most important properties of the discrete fractional Fisher's equation, namely, the existence and the uniqueness of positive and bounded solutions. The cornerstone of the proof is the concept of Minkowski matrices, and we recall this notion and its main properties in that section. This note closes with a section of concluding remarks and directions of future research.

\section{Continuous Motivation}

Throughout, we will assume that $a, b \in \mathbb{R}$ satisfy $a<b$ and that $\kappa, \lambda, K$, and $T$ are positive numbers. Let $\phi:[a, b] \rightarrow \mathbb{R}$ and $\psi_{1}, \psi_{2}:[0, T] \rightarrow \mathbb{R}$ be continuously differentiable functions whose ranges are subsets of $[0, K]$. Assume additionally that the compatibility conditions $\phi(a)=\psi_{1}(0)$ and $\phi(b)=$ $\psi_{2}(0)$ are satisfied. Let $1<\alpha<2$ and define $\Omega=(a, b) \times(0, T)$. In this work, we will suppose that $u: \bar{\Omega} \rightarrow \mathbb{R}$ is a sufficiently smooth function that satisfies the initial-boundary-value problem

$$
\begin{aligned}
& \frac{\partial u}{\partial t}(x, t)-\kappa \frac{\partial^{\alpha} u}{\partial|x|^{\alpha}}(x, t)=\lambda u(x, t)\left(1-\frac{u(x, t)}{K}\right), \\
& \text { such that } \begin{cases}u(x, 0)=\phi(x), & \forall x \in(a, b), \\
u(a, t)=\psi_{1}(t), & \forall t \in(0, T), \\
u(b, t)=\psi_{2}(t), & \forall t \in(0, T),\end{cases}
\end{aligned}
$$

for each $(x, t) \in \Omega$.
For the sake of convenience, we will define $u(x, t)=0$ for each $x \in(-\infty, a) \cup(b, \infty)$ and each $t \in[0, T]$. Using this convention, the space-fractional operator in (1) is the Riesz fractional derivative of order $\alpha$, which is given by

$$
\begin{aligned}
& \frac{\partial^{\alpha} u}{\partial|x|^{\alpha}}(x, t) \\
& \quad=\frac{-1}{2 \cos (\pi \alpha / 2) \Gamma(2-\alpha)} \frac{d^{2}}{d x^{2}} \int_{-\infty}^{\infty} \frac{u(\xi, t)}{|x-\xi|^{\alpha-1}} d \xi,
\end{aligned}
$$

for each $(x, t) \in \Omega$. Here, $\Gamma$ is the gamma function, which is defined in $\mathbb{R} \backslash\{n:-n \in \mathbb{N}$ or $n=0\}$ by

$$
\Gamma(z)=\int_{0}^{\infty} s^{z-1} e^{-s} d s
$$

Note that the partial differential equation of (1) is actually a space-fractional extension of the model from population dynamics investigated simultaneously and independently by Fisher [9] and Kolmogorov et al. [10] in 1937.

In this work, we follow a fractional difference approach to approximate the solutions of (1) and use fractional centered differences to approximate Riesz space-fractional derivatives. For any function $f: \mathbb{R} \rightarrow \mathbb{R}$, any $h>0$, and any $\alpha>-1$, the fractional centered difference of order $\alpha$ of $f$ at the point $x$ is defined as

$$
\Delta_{h}^{\alpha} f(x)=\sum_{k=-\infty}^{\infty} g_{k}^{\alpha} f(x-k h), \quad \forall x \in \mathbb{R},
$$

where

$$
g_{k}^{\alpha}=\frac{(-1)^{k} \Gamma(\alpha+1)}{\Gamma(\alpha / 2-k+1) \Gamma(\alpha / 2+k+1)}, \quad \forall k \in \mathbb{Z} .
$$

In the case that $1<\alpha \leq 2$, the fractional centered differences satisfy (see [23])

$$
\lim _{h \rightarrow 0} \frac{-1}{h^{\alpha}} \Delta_{h}^{\alpha} f(x)=\frac{\partial^{\alpha} f}{\partial|x|^{\alpha}}(x), \quad \forall x \in \mathbb{R} .
$$

Lemma 1 (see [24]). If $1<\alpha<2$, then the coefficients $\left(g_{k}^{\alpha}\right)_{k=-\infty}^{\infty}$ satisfy
(a) $g_{0}^{\alpha} \geq 0$,
(b) $g_{k}^{\alpha}=g_{-k}^{\alpha}<0$ for all $k \geq 1$,
(c) $\sum_{k=-\infty}^{\infty} g_{k}^{\alpha}=0$.

Lemma 2 (see [24]). Let $f \in \mathscr{C}^{5}(\mathbb{R})$ and assume that all its derivatives up to order five belong to $L_{1}(\mathbb{R})$. If $1<\alpha<2$, then

$$
-\frac{\Delta_{h}^{\alpha} f(x)}{h^{\alpha}}=\frac{\partial^{\alpha} f(x)}{\partial|x|^{\alpha}}+\mathcal{O}\left(h^{2}\right) .
$$

\section{Discrete Population Model}

For the remainder of this work, we assume that $M, N \in \mathbb{N}$ and use partition norms $h=(b-a) / M$ and $\tau=T / N$ 
for the intervals $[a, b]$ and $[0, T]$, respectively. For each $j \in$ $\{0,1, \ldots, M\}$ and each $n \in\{0,1, \ldots, N\}$, we define

$$
\begin{aligned}
& x_{j}=a+j h, \\
& t_{n}=n \tau, \\
& u_{j}^{n}=u\left(x_{j}, t_{n}\right) .
\end{aligned}
$$

Under these circumstances, note that

$$
\begin{aligned}
\frac{\partial^{\alpha} u}{\partial|x|^{\alpha}}\left(x_{j}, t_{n}\right) & =-\frac{1}{h^{\alpha}} \sum_{k=j-M}^{j} g_{k}^{\alpha} u_{j-k}^{n}+\mathcal{O}\left(h^{2}\right) \\
& =-\frac{1}{h^{\alpha}} \sum_{k=0}^{M} g_{j-k}^{\alpha} u_{k}^{n}+\mathcal{O}\left(h^{2}\right),
\end{aligned}
$$

for each $j \in\{1, \ldots, M-1\}$ and each $n \in\{1, \ldots, N-1\}$. In the following, we will consider the discrete operators

$$
\begin{aligned}
\delta_{t} u_{j}^{n} & =\frac{u_{j}^{n+1}-u_{j}^{n}}{\tau}, \\
\Delta_{h}^{\alpha} u_{j}^{n} & =-\frac{1}{h^{\alpha}} \sum_{k=0}^{M} g_{j-k}^{\alpha} u_{k}^{n} .
\end{aligned}
$$

Using this nomenclature, the fully discrete population model used in this work to approximate the solutions of (1) is given by the discrete system of equations

$$
\begin{gathered}
\delta_{t} u_{j}^{n}-\kappa \Delta_{h}^{\alpha} u_{j}^{n+1}=\lambda u_{j}^{n+1}\left(1-\frac{u_{j}^{n}}{K}\right), \\
\text { such that } \begin{cases}u_{j}^{0}=\phi\left(x_{j}\right), & \forall j \in\{0,1, \ldots, M\}, \\
u_{0}^{n}=f(a), & \forall n \in\{0,1, \ldots, N\}, \\
u_{M}^{n}=g(b), & \forall n \in\{0,1, \ldots, N\},\end{cases}
\end{gathered}
$$

for each $j \in\{1, \ldots, M-1\}$ and $n \in\{1, \ldots, N-1\}$. For convenience, Figure 1 shows the stencil of this system.

Remark 3.

(i) Observe that when $\alpha=2$, then $\Delta_{h}^{\alpha}$ becomes the classical discrete Laplace operator.

(ii) Also, note that the fully discrete fractional model (11) is computationally an implicit two-step discretization of the continuous system (1).

Note that the discrete model (11) has a convenient vector representation. To describe it, let us consider the mesh

$$
\mathscr{R}_{h}=\left\{x_{j}=a+j h: j=0,1, \ldots, M\right\},
$$

and let $\mathscr{V}_{h}$ be the real vector space of all grid functions on $\mathscr{R}_{h}$, that is, real functions $v$ defined on $\mathscr{R}_{h}$. For each $v \in \mathscr{V}_{h}$ and each $j \in\{0,1, \ldots, M\}$, we convey that $v_{j}=v\left(x_{j}\right)$. For each $n \in$ $\{0,1, \ldots, N\}$, let $u^{n} \in \mathscr{V}_{h}$ be the vector whose components are $u_{j}^{n}$ with $j \in\{0,1, \ldots, M\}$.

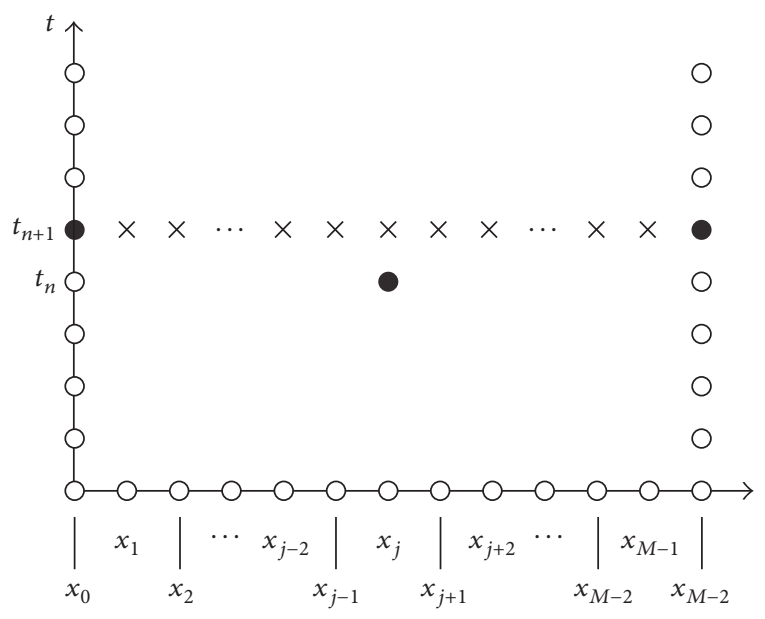

FIGURE 1: Stencil of the fully discrete model (11). The black circles represent the known solutions at the time $t_{n}$ and at the boundary, while the crosses denote the unknown approximations at the time $t_{n+1}$.

In what follows, we let $R=\kappa \tau / h^{\alpha}$ and define

$$
\begin{aligned}
& \widehat{g}_{j-k}^{\alpha}=R g_{j-k}^{\alpha}, \quad \forall k \in\{0,1, \ldots, M\} \backslash\{j\}, \\
& \widehat{g}_{0, j}^{\alpha, u^{n}}=1+R g_{0}^{\alpha}-\lambda \tau\left(1-\frac{u_{j}^{n}}{K}\right) .
\end{aligned}
$$

For the sake of briefness, in the following, we will obviate the dependence of $\widehat{g}_{0, j}^{\alpha, u^{n}}$ on $j$ and $n$. Recall from Lemma 1 that $g_{k}^{\alpha}=g_{-k}^{\alpha}$ for each $k \geq 1$. For each $n \in\{0,1, \ldots, N-1\}$, define the matrix $A_{u^{n}}$ of size $(M+1) \times(M+1)$ and the $(M+1)$ dimensional real vectors $\widehat{u}^{n}$ and $\Phi$ by

$$
\begin{aligned}
A_{u^{n}} & =\left(\begin{array}{cccccc}
1 & 0 & 0 & \cdots & 0 & 0 \\
\widehat{g}_{1}^{\alpha} & \widehat{g}_{0}^{\alpha} & \widehat{g}_{1}^{\alpha} & \cdots & \widehat{g}_{M-2}^{\alpha} & \widehat{g}_{M-1}^{\alpha} \\
\widehat{g}_{2}^{\alpha} & \widehat{g}_{1}^{\alpha} & \widehat{g}_{0}^{\alpha} & \cdots & \widehat{g}_{M-3}^{\alpha} & \widehat{g}_{M-2}^{\alpha} \\
\vdots & \vdots & \vdots & \ddots & \vdots & \vdots \\
\widehat{g}_{M-1}^{\alpha} & \widehat{g}_{M-2}^{\alpha} & \widehat{g}_{M-3}^{\alpha} & \cdots & \widehat{g}_{0}^{\alpha} & \widehat{g}_{1}^{\alpha} \\
0 & 0 & 0 & \cdots & 0 & 1
\end{array}\right), \\
\widehat{u}^{n} & =\left(f\left(t_{n+1}\right), u_{1}^{n}, u_{2}^{n}, \ldots, u_{M-1}^{n}, g\left(t_{n+1}\right)\right), \\
\Phi_{u} & =\left(\phi\left(x_{0}\right), \phi\left(x_{1}\right), \ldots, \phi\left(x_{M-1}\right), \phi\left(x_{M}\right)\right) .
\end{aligned}
$$

Under these circumstances, the fully discrete population model (11) with the set of initial-boundary data $(\Phi, f, g)$ may be expressed equivalently as the vector problem

$$
A_{u^{n}} u^{n+1}=\widehat{u}^{n}, \quad \forall n \in\{0,1, \ldots, N-1\},
$$

such that $u^{0}=\Phi_{u}$.

\section{Properties}

For convenience, we denote the ranges of $\phi, f$, and $g$ by $\mathscr{R}_{\phi}, \mathscr{R}_{f}$, and $\mathscr{R}_{g}$, respectively. Recall that a matrix $A$ is 
nonnegative if every entry of $A$ is a nonnegative number, a fact that is denoted by $A \geq 0$. If $s$ is any real number, we will say that $A$ is bounded from above by $s$ if every entry of $A$ is less than or equal to $s$, a fact that will be represented by $A \leq s$. Obviously, an $n$-dimensional real vector $v$ satisfies $v \leq s$ if and only if $s e-v \geq 0$, where $e$ is the $n$-dimensional vector whose components are all equal to 1 .

A real square matrix $A$ is a $Z$-matrix if all its off-diagonal entries are less than or equal to zero. We say that $A$ is a Minkowski matrix if the following are satisfied:

(i) $A$ is a $Z$-matrix.

(ii) All the diagonal entries of $A$ are positive.

(iii) There exists a diagonal matrix $D$ with positive diagonal elements, such that $A D$ is strictly diagonally dominant.

Obviously, if $A$ is strictly diagonally dominant, then condition (iii) is trivially satisfied with $D$ equal to the identity.

The following result is part of the standard literature.

Lemma 4 (Fujimoto and Ranade [25]). Every Minkowski matrix is nonsingular, and its inverse is a positive matrix.

Lemma 5. Let $n \in\{0,1, \ldots, N-1\}$ and suppose $\mathscr{R}_{f}, \mathscr{R}_{g} \subseteq$ $[0, K]$ and $\lambda \tau<1$. If $0 \leq u^{n} \leq K$, then $A_{u^{n}}$ is a Minkowski matrix.

Proof. The off-diagonal entries of $A_{u^{n}}$ are 0 or of the form $\widehat{g}_{j-k}^{\alpha}$ for $j \in\{1, \ldots, M-1\}, k \in\{0,1, \ldots, M\}$, and $k \neq j$, so they are nonpositive by Lemma 1(b). On the other hand, Lemma 1(a) guarantees that $g_{0}^{\alpha} \geq 0$ and the hypotheses yield that

$$
0 \leq 1-\frac{u_{j}^{n}}{K} \leq 1 \text {, }
$$

whence

$$
\widehat{g}_{0}^{\alpha} \geq 1-\lambda \tau\left(1-\frac{u_{j}^{n}}{K}\right) \geq 1-\lambda \tau>0
$$

follows. Finally, note that Lemma 1(c) implies that

$$
\begin{aligned}
\sum_{\substack{k=0 \\
k \neq j}}^{M}\left|\widehat{g}_{j-k}^{\alpha}\right| & =R \sum_{\substack{k=0 \\
k \neq j}}^{M}\left|g_{j-k}^{\alpha}\right| \leq R \sum_{\substack{k=-\infty \\
k \neq 0}}^{\infty}\left|g_{k}^{\alpha}\right|=R g_{0}^{\alpha} \\
& <1+R g_{0}^{\alpha}-\lambda t\left(1-\frac{u_{j}^{n}}{K}\right)=\widehat{g}_{0}^{\alpha}
\end{aligned}
$$

for each $j \in\{1, \ldots, M-1\}$. So, $A_{u^{n}}$ is strictly diagonally dominant, and we conclude that it is a Minkowski matrix.

We provide an additional algebraic property of the matrices $A_{u^{n}}$. To that end, consider $V=\mathbb{R}^{M+1}$ as a real vector space with the usual operations and denote the vector space of linear operators on $\mathbb{R}^{M+1}$ by $\operatorname{hom}_{\mathbb{R}}(V, V)$.
Lemma 6. Let $\lambda \tau<1$ and let $A: V \rightarrow \operatorname{hom}_{\mathbb{R}}(V, V)$ be given by

$$
A(u)(w)=A_{u} w, \quad \forall u, w \in V .
$$

Then, $A$ is a linear transformation such that, for each $u, v, w \in$ $V$, if $0 \leq u \leq v \leq K$ and $w \geq 0$, then $0 \leq A_{u}(w) \leq A_{v}(w)$.

Proof. Under the hypotheses, it is clear that $\widehat{g}_{0, j}^{\alpha, u} \leq \widehat{g}_{0, j}^{\alpha, v}$. As a consequence,

$$
A_{u}(w)=\left(\begin{array}{c}
w_{0} \\
\widehat{g}_{0,1}^{\alpha, u} w_{1}+\sum_{k=0}^{M} \widehat{g}_{1-k}^{\alpha} w_{k} \\
\hat{g}_{0,2}^{\alpha, u} w_{2}+\sum_{k=0}^{M} \widehat{g}_{2-k}^{\alpha} w_{k} \\
\vdots \\
\widehat{g}_{0, M-1}^{\alpha, u} w_{M-1}+\sum_{k=0}^{M} \widehat{g}_{M-1-k}^{\alpha} w_{k} \\
w_{M}^{n+1} \\
w_{0}^{M} \\
\hat{g}_{0,1}^{\alpha, v} w_{1}+\sum_{k=0}^{M} \widehat{g}_{1-k}^{\alpha} w_{k} \\
\hat{g}_{0,2}^{\alpha, v} w_{2}+\sum_{k=0}^{M} \widehat{g}_{2-k}^{\alpha} w_{k} \\
\vdots \\
\hat{g}_{0, M-1}^{\alpha, v} w_{M-1}+\sum_{k=0}^{M} \widehat{g}_{M-1-k}^{\alpha} w_{k} \\
w_{M}
\end{array}\right)
$$

As a consequence, $A_{u}(w) \leq A_{v}(w)$. Moreover, it is obvious that $A_{u}(w) \geq 0$.

The following is the most important result of this work.

Theorem 7 (positivity and boundedness). Suppose that $\mathscr{R}_{\phi}, \mathscr{R}_{f}, \mathscr{R}_{g} \in[0, K]$ and $\lambda \tau<1$. Then, there exists a unique solution $\left(u^{n}\right)_{n=0}^{N}$ of (11) satisfying $0 \leq u^{n} \leq K$ for each $n \epsilon$ $\{0,1, \ldots, N\}$.

Proof. Note that $u^{0}$ satisfies the conclusion by hypothesis, so assume that it is true for some $n \in\{0,1, \ldots, N-1\}$. By Lemmas 4 and 5, the matrix $A_{u^{n}}$ is nonsingular and the entries of its inverse are positive numbers. Moreover, $\widehat{u}^{n} \geq$ 0 by the induction hypothesis and the assumptions on the boundary conditions. It follows that

$$
u^{n+1}=\left(A_{u^{n}}\right)^{-1} \widehat{u}^{n} \geq 0,
$$

and we only need to show that $u^{n+1} \leq K$ or, equivalently, that $v^{n+1}=K e-u^{n+1} \geq 0$, where $e$ is the vector of the same 
dimension as $u^{n+1}$, all of whose entries are equal to 1 . Note that the recursive identity in (15) is equivalent to $A_{u^{n}} v^{n+1}=$ $b^{n}$, where

$$
b^{n}=\left(\begin{array}{c}
K-f\left(t_{n+1}\right) \\
\sum_{k=0}^{M} K \widehat{g}_{1-k}^{\alpha}-u_{1}^{n} \\
\sum_{k=0}^{M} K \widehat{g}_{2-k}^{\alpha}-u_{2}^{n} \\
\vdots \\
\sum_{k=0}^{M} K \widehat{g}_{M-1-k}^{\alpha}-u_{M-1}^{n} \\
K-g\left(t_{n+1}\right)
\end{array}\right) \text {. }
$$

Clearly, the first and the last components of $b^{n}$ are nonnegative. Meanwhile, after a simplification, one may readily check that the $(j+1)$ st component of $b^{n}$ is of the form

$$
\begin{aligned}
& b_{j}^{n}=\sum_{k=0}^{M} K \widehat{g}_{j-k}^{\alpha}-u_{j}^{n} \\
& =K R\left(g_{0}^{\alpha}+\sum_{\substack{k=0 \\
k \neq j}}^{M} g_{j-k}^{\alpha}\right)+\left(K-u_{j}^{n}\right)(1-\lambda \tau) \geq 0,
\end{aligned}
$$

for each $j \in\{1, \ldots, M-1\}$. It follows that $b^{n} \geq 0$, and the fact that $A_{u^{n}}$ is a Minkowski matrix yields that $v^{n+1} \geq 0$ or, equivalently, that $u^{n+1} \leq K$. The result follows now by induction.

From a practical point of view, Theorem 7 establishes that the fully discrete model (11) possesses unique solutions which may be interpreted as population densities when $K=1$. Indeed, the theorem guarantees that, for any initial-boundary data which are nonnegative and bounded from above by $K$, there exists a unique solution of (11) with the same properties of nonnegativity and boundedness. In that sense, our fully discrete model may be an ideal paradigm to describe the dynamics of variables measured in absolute scales which are normalized with respect to some maximum value. These features are in agreement also with respect to nonfractional models of science and engineering such as Fisher's equation and various of its generalizations.

The following example is presented to illustrate the capability of the fully discrete system (11) to preserve the nonnegativity and the boundedness of solutions.

Example 8. Fix the parameters $a=-200, b=200, \kappa=\lambda=1$, and $K=1.2$, let $\psi_{1}(t)=\psi_{2}(t)=0$ for each $t>0$, and define

$$
\phi(x)= \begin{cases}0.1, & \text { if } x=0 \\ 0, & \text { otherwise }\end{cases}
$$

Computationally, let $h=1, \tau=0.01$. Under these circumstances, Figure 2 provides snapshots of the solutions of the fully discrete model (11) at various times for several values of $\alpha$, namely, 2 (solid), 1.9 (dashed), 1.8 (dasheddotted), and 1.7 (dotted). The graphs show that the diffusion increases when $\alpha$ decreases. This is a typical behavior of partial differential equations with fractional diffusion and is in full agreement with results obtained in the literature [26].

Before we close this section, it is important to note that the matrices $A_{u}$ are Hermitian and positive-definite under the hypotheses of the theorem, so they have Cholesky factorizations of the form $A=L L^{*}$, where $L$ is a lower triangular matrix of the same size as $A$ and $L^{*}$ is its conjugate transpose. This means in particular that the discrete population model (11) may be efficiently implemented using the Cholesky decomposition method. It is important to point out that the fully discrete model (11) has been implemented computationally using this technique. Some of the results are shown in Example 8. We have conducted more numerical experiments which are not presented here in view that they also confirm the preservation of the properties of positivity and boundedness of the solutions

\section{Conclusions and Perspectives}

In this note, we considered a fully discrete model associated with a diffusion-reaction partial differential equation with Riesz space-fractional derivatives which generalizes Fisher's equation from population dynamics and nuclear physics. The spatial derivative orders are in the interval $(1,2)$, and the spatial discretization of the model uses fractional centered differences, which are consistent discretizations of the Riesz fractional derivatives. The fully discrete population model is implicit and linear, and it can be represented in vector form using an $M$-matrix under suitable parameter conditions. As a consequence, the scheme is always solvable, and the properties of $M$-matrices guarantee the positivity and the boundedness of the solutions. We calculated the solution of some initial-boundary-value problems governed by the discrete fractional Fisher's equation through an implementation of the Cholesky decomposition algorithm. The results are not provided in this work, but they confirm the capacity of the model to preserve the positivity and the boundedness when the initial-boundary data satisfy those properties.

Various avenues of research open after the conclusion of this manuscript. For example, it would be interesting to determine whether theorems on the existence and the uniqueness of positive and bounded solutions for more general discrete fractional systems can be established. There are many generalizations of Fisher's equation in the context of population dynamics, and various approaches may be followed in order to provide fractional extensions for those models. An interesting question that arises is whether those discrete systems accept solutions with the characteristics of interest. In that sense, the present note is an affirmative step toward the structural analysis of discrete fractional systems arising in population dynamics. Once we have established relevant theorems on the solutions of the discrete fractional Fisher's equation, another interesting avenue of research is the numerical analysis of structure-preserving methods to 


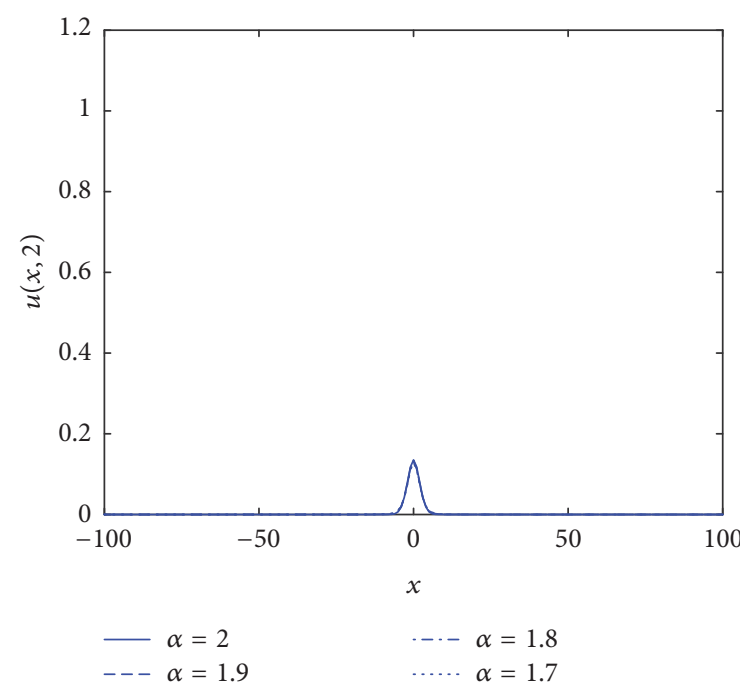

(a)

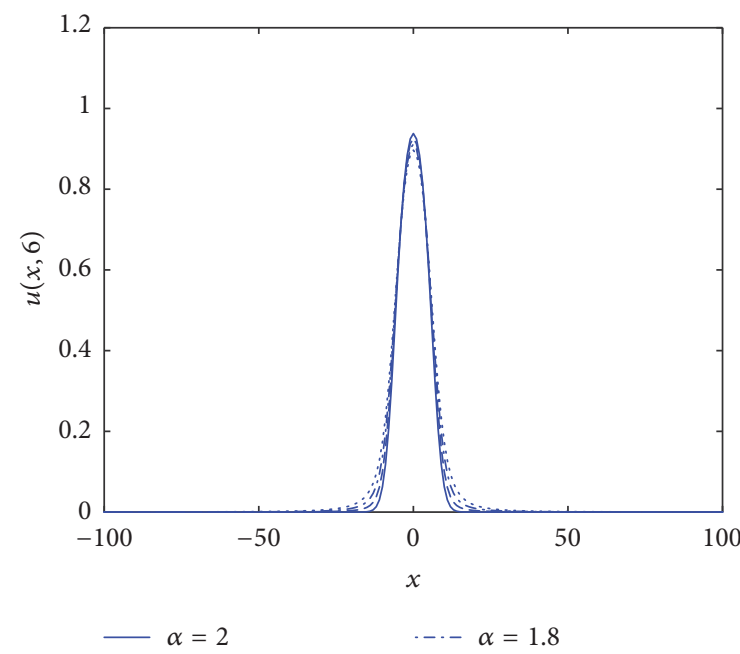

$---\alpha=1.9$

(c)

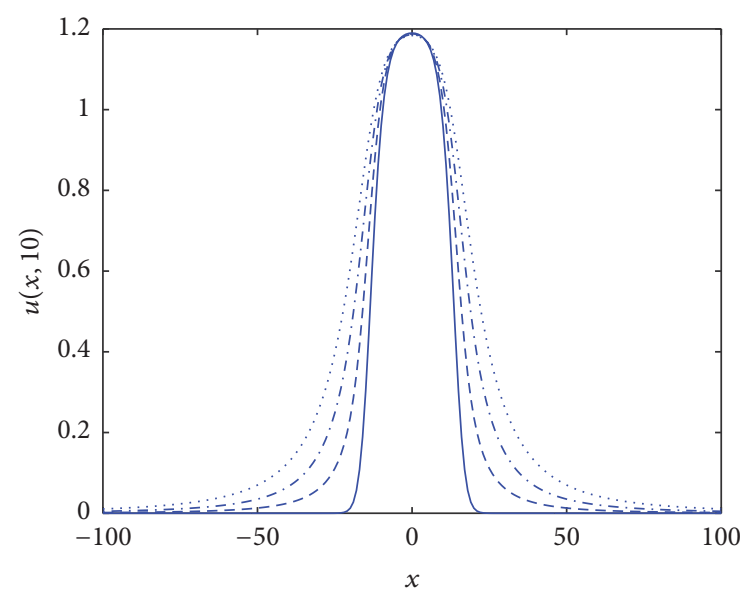

$-\alpha=2$

$---\alpha=1.9$

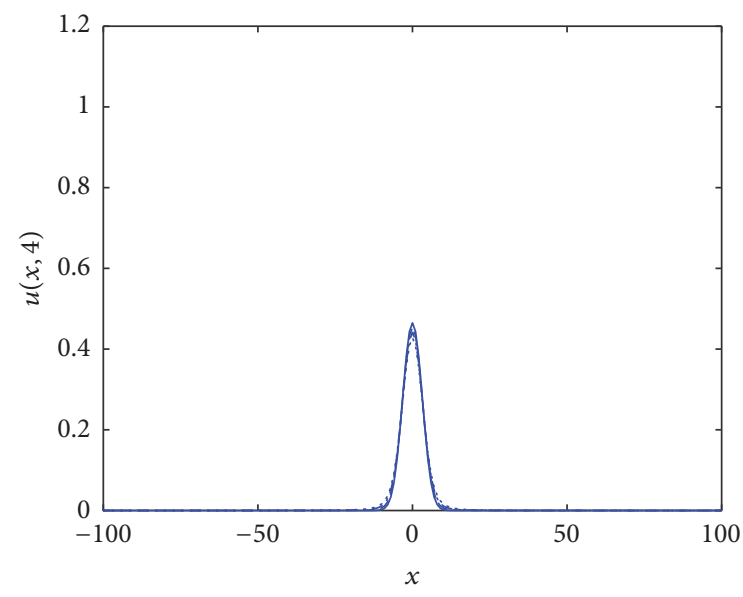

$\alpha=2$

$--\alpha=1.9$

$\cdots \alpha=1.8$

… $\alpha=1.7$

(b)

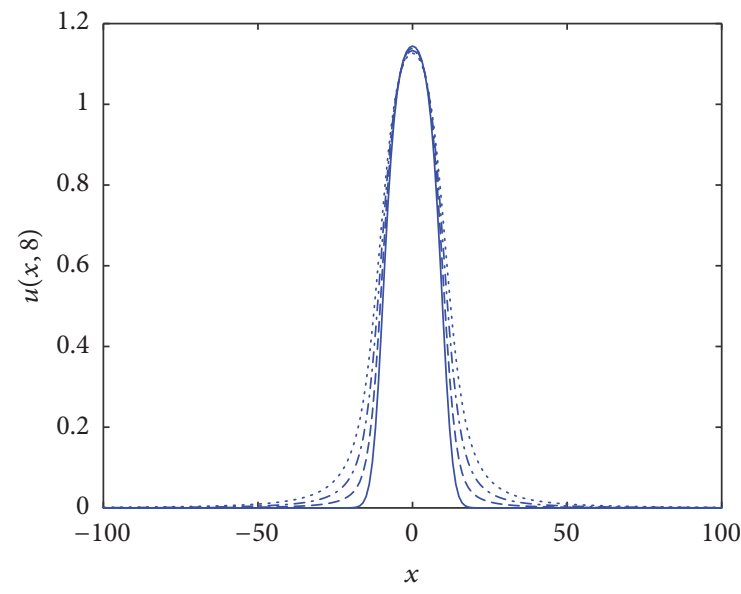

$-\alpha=2$

$---\alpha=1.9$

-.- $\alpha=1.8$

… $\alpha=1.7$

(d)

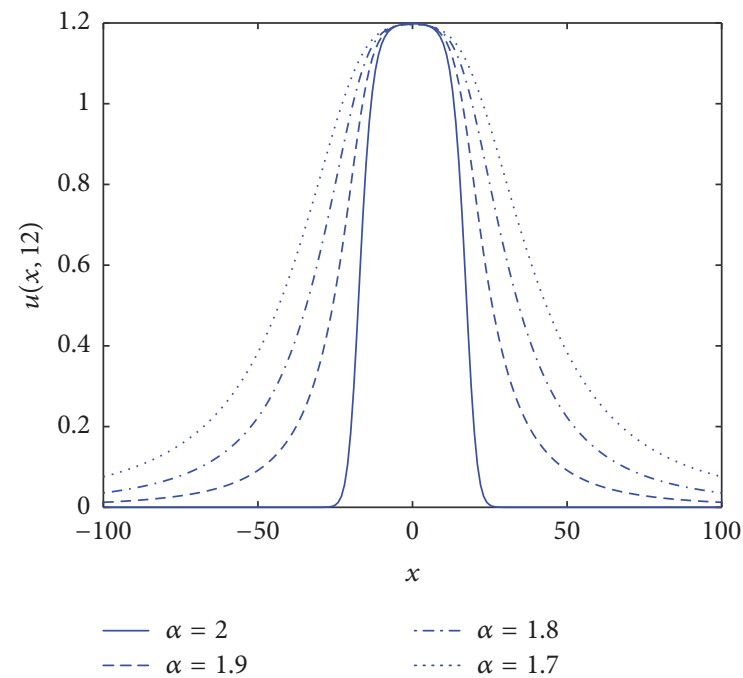

(f)

FIGURE 2: Graph of the solution of the fully discrete model (11) over the spatial domain $(-200,200)$ with $\kappa=\lambda=1, K=1.2$, homogeneous Dirichlet boundary data, and initial profile given by (24). Computationally, we used $h=1$ and $\tau=0.01$. Various derivative orders were used, namely, $\alpha=2$ (solid), $\alpha=1.9$ (dashed), $\alpha=1.8$ (dashed-dotted), and $\alpha=1.7$ (dotted). The solutions were calculated at the times (a) $t=2$, (b) $t=4$, (c) $t=6$, (d) $t=8$, (e) $t=10$, and (f) $t=12$. For convenience, the graphs have been plotted on the domain $(-100,100)$. 
approximate continuous fractional models. The literature already has some relevant properties on the consistency of fractional centered differences, but the design of structurepreserving methods for fractional partial differential equations is still an area that is yet to be explored. The topic is outside the scope of this journal, but it is a problem that deserves a thorough numerical investigation.

\section{Conflicts of Interest}

The author declares that there are no conflicts of interest regarding the publication of this paper.

\section{References}

[1] A.-M. Yang, J. Li, H. M. Srivastava, G.-N. Xie, and X.-J. Yang, "Local fractional Laplace variational iteration method for solving linear partial differential equations with local fractional derivative," Discrete Dynamics in Nature and Society. An International Multidisciplinary Research and Review Journal, vol. 2014, Article ID 365981, 8 pages, 2014.

[2] X. Sha, H. Ge, and J. Xin, "On the existence and stability of standing waves for 2-coupled nonlinear fractional Schrödinger system," Discrete Dynamics in Nature and Society. An International Multidisciplinary Research and Review Journal, vol. 2015, Article ID 427487, 8 pages, 2015.

[3] V. E. Tarasov and E. C. Aifantis, "Non-standard extensions of gradient elasticity: fractional non-locality, memory and fractality," Communications in Nonlinear Science and Numerical Simulation, vol. 22, no. 1-3, pp. 197-227, 2015.

[4] S. R. Grace, T. S. Hassan, S. Sun, and E. Akin, "Qualitative Analysis on Differential, Fractional Differential, and Dynamic Equations and Related Topics," Discrete Dynamics in Nature and Society, vol. 2016, Article ID 3590319, 2 pages, 2016.

[5] V. E. Tarasov and G. M. Zaslavsky, "Conservation laws and Hamilton's equations for systems with long-range interaction and memory," Communications in Nonlinear Science and Numerical Simulation, vol. 13, no. 9, pp. 1860-1878, 2008.

[6] W. Lv, "Existence and uniqueness of solutions for a discrete fractional mixed type sum-difference equation boundary value problem," Discrete Dynamics in Nature and Society. An International Multidisciplinary Research and Review Journal, vol. 2015, Article ID 376261, 10 pages, 2015.

[7] X. Li, G. Zhang, and Y. Wang, "Existence and uniqueness of positive solitons for a second-order difference equation," Discrete Dynamics in Nature and Society. An International Multidisciplinary Research and Review Journal, vol. 2014, Article ID 503496, 8 pages, 2014.

[8] R. Dahal, D. Duncan, and C. . Goodrich, "Systems of semipositone discrete fractional boundary value problems," Journal of Difference Equations and Applications, vol. 20, no. 3, pp. 473491, 2014.

[9] R. A. Fisher, "The, wave of advance of advantageous genes," Annals of Eugenics, vol. 7, no. 4, pp. 355-369, 1937.

[10] A. N. Kolmogorov, I. G. Petrovskii, and N. S. Piskunov, "A study of the equation of diffusion with increase in the quantity of matter, and its application to a biological problem," Moscow University Bulletin of Mathematics, vol. 1, no. 7, pp. 1-26, 1937.

[11] G. Lu, "Bifurcation analysis of gene propagation model governed by reaction-diffusion equations," Discrete Dynamics in
Nature and Society. An International Multidisciplinary Research and Review Journal, vol. 2016, Article ID 9840297, 9 pages, 2016.

[12] W. E. Kastenberg, P. L. Chambr $\Theta$, and P. L. Chambré, "On the stability of nonlinear space-dependent reactor kinetics," Nuclear Science and Engineering, vol. 31, no. 1, pp. 67-79, 1968.

[13] S. T. Mohyud-Din, A. Ali, and B. Bin-Mohsin, "On biological population model of fractional order," International Journal of Biomathematics, vol. 9, no. 5, Article ID 1650070, 13 pages, 2016.

[14] D. Stan and J. L. Vázquez, "The Fisher-KPP equation with nonlinear fractional diffusion," SIAM Journal on Mathematical Analysis, vol. 46, no. 5, pp. 3241-3276, 2014.

[15] A. Szafran'ska and J. E. Macas-Daz, "On the convergence of a finite-difference discretization à la Mickens of the generalized Burgers-Huxley equation," Journal of Difference Equations and Applications, vol. 20, no. 10, pp. 1444-1451, 2014.

[16] S. Tomasiello, "Stability and accuracy of the iterative differential quadrature method," International Journal for Numerical Methods in Engineering, vol. 58, no. 9, pp. 1277-1296, 2003.

[17] S. Tomasiello, "Numerical solutions of the Burgers-Huxley equation by the IDQ method," International Journal of Computer Mathematics, vol. 87, no. 1-3, pp. 129-140, 2010.

[18] Z. Liu, S. Lv, and X. Li, "Legendre collocation spectral method for solving space fractional nonlinear Fisher's equation," in Asian Simulation Conference, vol. 643 of Communications in Computer and Information Science, pp. 245-252, Springer, Beijing, China.

[19] X. Zhang, Y. He, L. Wei, B. Tang, and S. Wang, "A fully discrete local discontinuous Galerkin method for one-dimensional time-fractional Fisher's equation," International Journal of Computer Mathematics, vol. 91, no. 9, pp. 2021-2038, 2014.

[20] Q. Yang, I. Turner, T. Moroney, and F. Liu, "A finite volume scheme with preconditioned Lanczos method for twodimensional space-fractional reaction-diffusion equations," Applied Mathematical Modelling. Simulation and Computation for Engineering and Environmental Systems, vol. 38, no. 15-16, pp. 3755-3762, 2014.

[21] M. H. Cherif, K. Belghaba, and D. Ziane, "Homotopy perturbation method for solving the fractional Fisher's equation," International Journal of Analysis and Applications, vol. 10, no. 1, pp. 9-16, 2016.

[22] R. E. Mickens, "Calculation of order $\epsilon$ dynamics for an NSFD discretization of a cube-root damped oscillator," Journal of Difference Equations and Applications, vol. 20, no. 5-6, pp. 826836, 2014.

[23] M. D. Ortigueira, "Riesz potential operators and inverses via fractional centred derivatives," International Journal of Mathematics and Mathematical Sciences, vol. 2006, Article ID 48391, 12 pages, 2006.

[24] C. Çelik and M. Duman, "Crank-Nicolson method for the fractional diffusion equation with the Riesz fractional derivative," Journal of Computational Physics, vol. 231, no. 4, pp. 1743-1750, 2012.

[25] T. Fujimoto and R. R. Ranade, "Two characterizations of inverse-positive matrices: the Hawkins-Simon condition and the Le Chatelier-Braun principle," Electronic Journal of Linear Algebra, vol. 11, pp. 59-65, 2004.

[26] B. Baeumer, M. Kovács, and M. M. Meerschaert, "Fractional reproduction-dispersal equations and heavy tail dispersal kernels," Bulletin of Mathematical Biology, vol. 69, no. 7, pp. 22812297, 2007. 


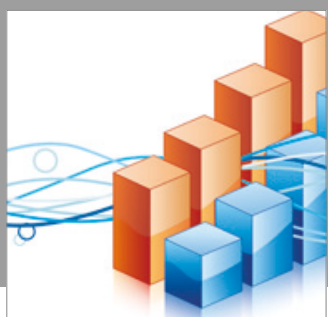

Advances in

Operations Research

vatersals

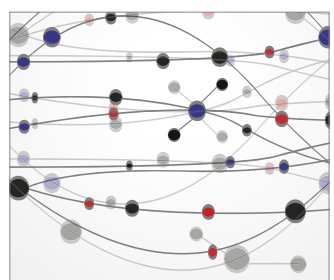

\section{The Scientific} World Journal
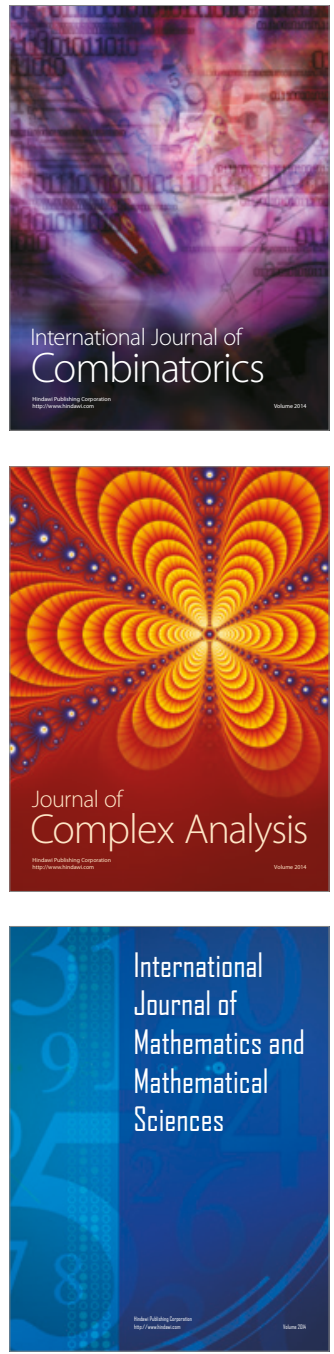
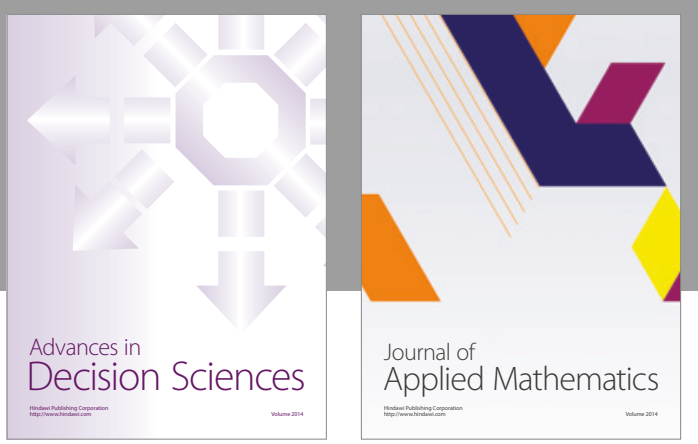

Algebra

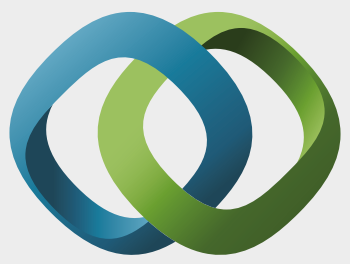

\section{Hindawi}

Submit your manuscripts at

https://www.hindawi.com
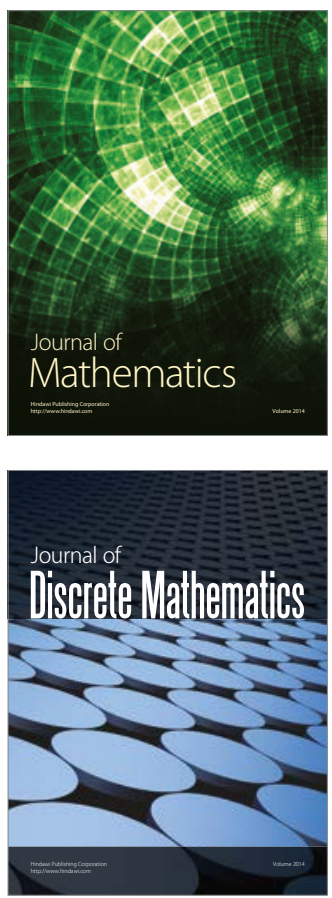

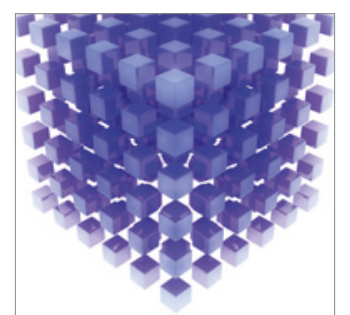

Mathematical Problems in Engineering
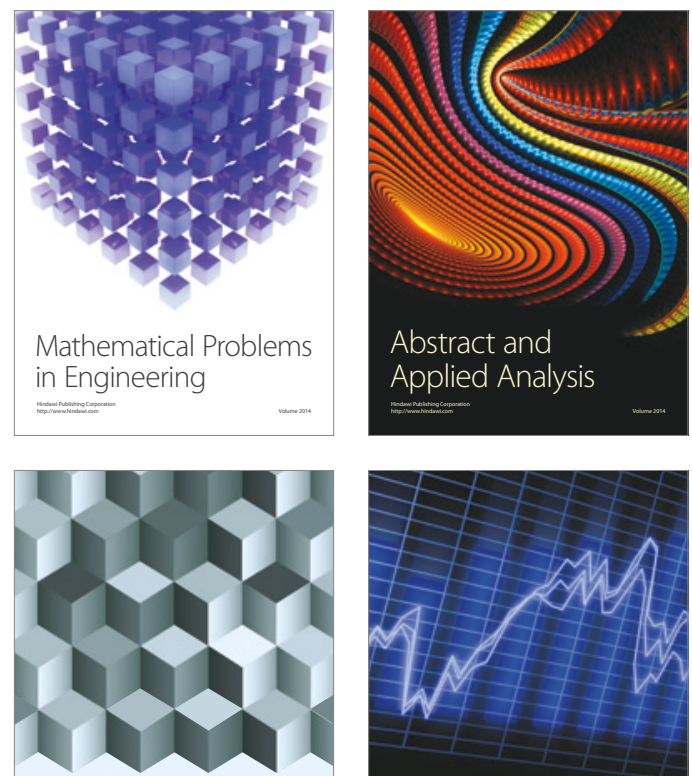

Journal of

Function Spaces

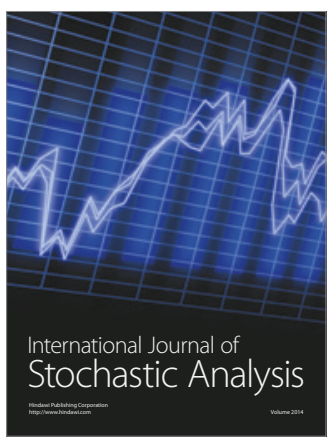

Probability and Statistics
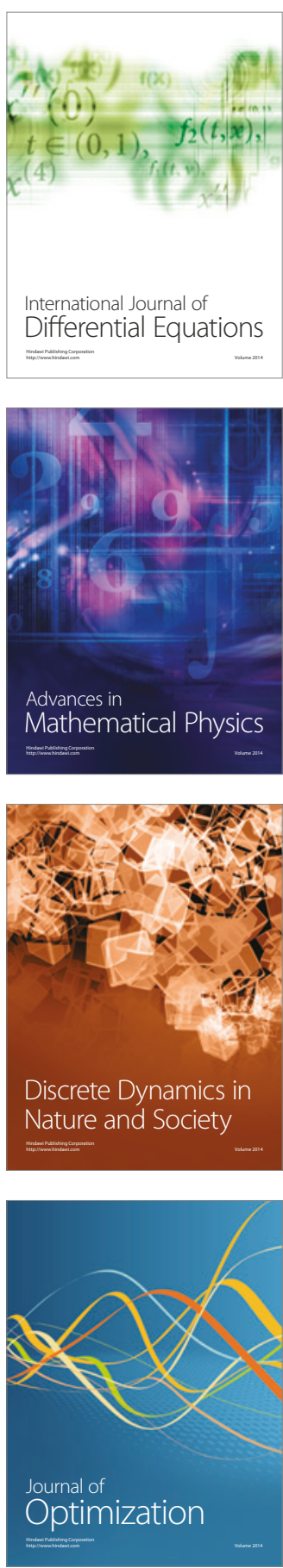